\title{
Electromagnetically Induced Magnetochiral Anisotropy in a Resonant Medium
}

\author{
V. A. Sautenkov, ${ }^{1,2}$ Y. V. Rostovtsev,${ }^{1}$ H. Chen,${ }^{1}$ P. Hsu, ${ }^{1}$ Girish S. Agarwal,,${ }^{3, *}$ and M. O. Scully ${ }^{1,4}$ \\ ${ }^{1}$ Institute for Quantum Studies and Department of Physics, Texas A\&M University, Texas 77843, USA \\ ${ }^{2}$ Lebedev Institute of Physics, Moscow 119991, Russia \\ ${ }^{3}$ Department of Physics, Oklahoma State University, Stillwater, Oklahoma 74078, USA \\ ${ }^{4}$ Department of Mechanical \& Aerospace Engineering, Princeton University, Princeton, New Jersey 08544, USA
}

(Received 16 January 2005; published 14 June 2005)

\begin{abstract}
Chirality has been extensively studied for well over a century, and its potential applications range from optics to chemistry, medicine, and biology. Ingenious experiments have been designed to measure this naturally small effect. Here we discuss the possibility of producing a medium having a large chiral effect by using the ideas of coherent control. The coherent fields resonant with appropriate transitions in atomic or molecular systems can be used to manipulate the optical properties of a medium. We demonstrate experimentally very large magnetochiral anisotropy by using electromagnetic fields in atomic $\mathrm{Rb}$ vapors.
\end{abstract}

DOI: 10.1103/PhysRevLett.94.233601

Atoms and molecules in electromagnetic fields can be considered a new state of matter that continues to attract a great deal of attention. Electromagnetic fields provide a method to manipulate the properties of matter such as absorption, dispersion, and a variety of nonlinear characteristics [1-4]. Similar to the fabrication of new materials, like photonic crystals or nanostructures, an applied coherent field allows one to improve the performance of devices and go beyond the limitations set by natural materials by themselves. In particular, it creates conditions for lasers to operate without population inversion between levels relevant for the lasing transition. Giant nonlinearities [1] and large refractive indexes [3] have been achieved. Giant nonlinearities may enable one to generate nonlinear signals using single photons [5]. The enhanced nonlinearity can also bring the light pulse to slow down considerably [6-8], and this is used for quantum information storage $[9,10]$.

Here we answer the question of whether it is possible to use the quantum coherent effects to enhance the effect of optical activity [11]. Optically active molecules are abundant in nature. In fact, the nearly exclusive utilization of one form of the optical antipode pair may be considered as a characteristic feature of living systems. Optical activity has always stimulated researchers' imaginations, as the substitution and modification of natural products is not only a challenge but also of high practical importance. It is important to control chirality or to create an environment that displays chirality in a controllable way, which can have applications in drug production, spin chemistry, etc. In this Letter we report the experimental observation of very large optically induced chirality in a Rb cell.

The chirality of a molecule is a symmetry property: either chiral molecules are asymmetric, i.e., they do not have any symmetry element, or they are symmetric, i.e., they have only axes of rotation. If an atomic or molecular system has spherical symmetry, then the transmission coefficient is the same for light propagating parallel to the magnetic field and for light propagating in the antiparallel direction with respect to the magnetic field. If a symmetry
PACS numbers: $32.80 . \mathrm{Qk}, 42.50 . \mathrm{Hz}, 42.65 . \mathrm{Dr}$

operation of reflection is performed on a chiral molecule, it is transformed into a different chiral system of equal energy and other nonvectorial properties. This molecule is called a mirror image, the optical antipode, or the enantiomer. Molecular systems that show chirality have an obvious or hidden screw property, or a handedness. In the case when the molecule has no symmetry, then there is the phenomenon of optical chirality. Arago's discovery in 1811 of natural optical activity in chiral crystals and Faraday's discovery in 1846 of magnetically induced optical activity have contributed much to our understanding of the wave nature of light and the electronic properties of molecules. Both effects are manifest as a rotation in the polarization of transmitted light: the former is due to the intrinsic properties of media that lack mirror symmetry, whereas the latter (which occurs in all materials) is due to magnetic-field-induced changes in the optical properties.

Recently, a new polarization-independent optical effect was discovered: magnetochiral anisotropy (MCA). The existence of this effect may be important in the context of fundamental interactions between light and matter, and in molecular spectroscopy, although the effect is generally very weak for naturally occurring systems [12-17]. In a recent paper Agarwal and Dasgupta [18] discovered that it is possible to produce very large MCA using electric dipole transitions and by applying a properly polarized coherent field so as to break the symmetry of the system. We refer to this type of MCA as electromagnetically induced magnetochiral anisotropy (EIMCA). We note that very interesting experiments on birefringence induced by laser fields in the absence of any magnetic field have been reported [19].

The idea of optically induced chirality is the following. First, consider a simplified model of the $\mathrm{Rb}$ atom, a threelevel atom [see Fig. 1]; the Hamiltonian of the atom in magnetic and optical fields is given by

$$
H=\hbar \omega_{+}|-\rangle\left\langle-\left|\hbar \omega_{-}\right|+\right\rangle\langle+|+\hbar \Omega|+\rangle\langle f|+\text { H.a. }
$$

where $|-\rangle \equiv|m=-1\rangle,|+\rangle \equiv|m=+1\rangle,|f\rangle \equiv \mid f, m=$ $0\rangle$, and $\omega_{-}, \omega_{+}$are the atomic states and their eigenvalues 


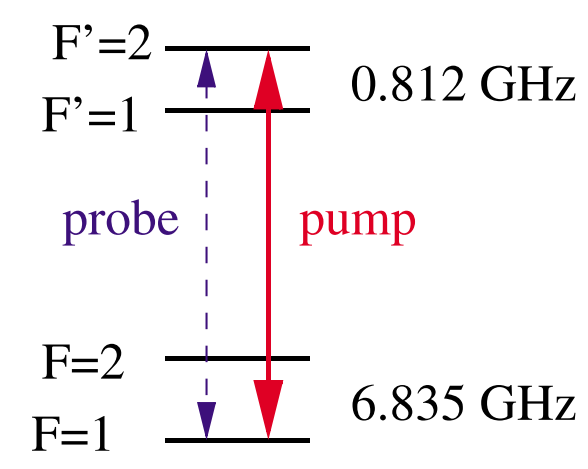

(a)

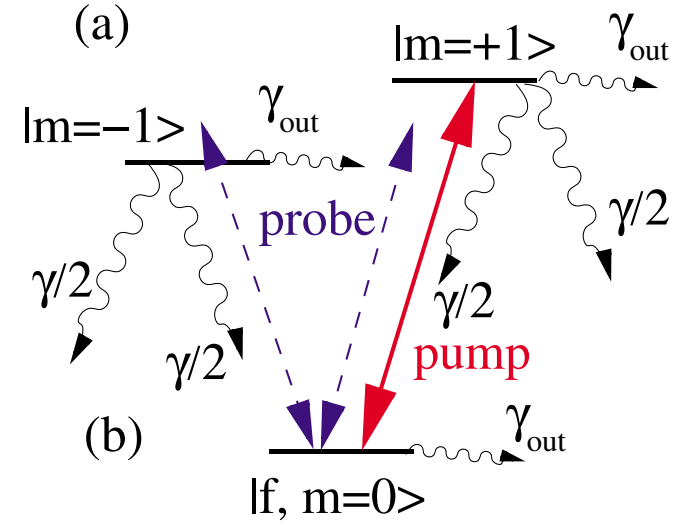

FIG. 1 (color online). (a) Rb level structure and configuration of laser fields. The pump field is circularly polarized; the probe field is not polarized and can be viewed as a noncoherent mixture of left and right circular polarized light. (b) A three-level model is used in our simulations. Relaxation of population $\gamma$ is shown by undulating arrows. The simple model also accounts for flight time broadening at rate $\gamma_{\text {out }}$ out of the system from all levels, and population relaxation from electronic excited states $|m=-1\rangle$ and $|m=+1\rangle$ to other than $|f, m=0\rangle$ levels of ground state manifold via spontaneous decay. Note that for our simulation we use a simplified three-level model; the real D1 line of Rb has three sets of similar three-level systems: $|m=-2\rangle \leftrightarrow \mid f, m=$ $-1\rangle \leftrightarrow|m=0\rangle,|m=0\rangle \leftrightarrow|f, m=+1\rangle \leftrightarrow|m=2\rangle$, and the one shown above.

in the magnetic field. The Hamiltonian has a symmetry with respect to a change in the direction of the magnetic field, namely, if $B \rightarrow-B$, then $|-\rangle \rightarrow|+\rangle,|-\rangle \rightarrow|+\rangle$ and $\omega_{+} \rightarrow \omega_{-}, \omega_{+} \rightarrow \omega_{-}$. The term coupled to an auxiliary field $\Omega$ breaks symmetry and introduces chirality in the system. Indeed, the susceptibility is given by

$$
\chi_{i j}(\omega, k, B, \Omega)=\tilde{\chi}_{i j}(\omega, \Omega)+\alpha_{i j l}(\omega, \Omega) k_{l}+\beta_{i j l}(\omega, \Omega) B_{l}
$$

$$
+\gamma_{i j l m}(\omega, \Omega) k_{l} B_{m}
$$

where $\alpha_{i j l}$ describes natural optical activity and $\beta_{i j l}$ describes magnetic optical activity; $\gamma_{i j l m}$ describes the magnetochiral effect; $\omega$ and $k$ are the frequency and the wave vector of the optical field. In the case of atomic vapors, $\gamma_{i j l m}$ is zero. Applying an external driving field $\Omega$ leads to magnetochirality via the last term.

The experiment that demonstrates the symmetry breaking effect is performed in rubidium vapor (level structure shown in Fig. 1(a)]. A schematic diagram of the experimental setup is shown in Fig. 2. Two external cavity diode lasers (ECDL) are used in our measurements. Radiation from the first ECDL used as a pump is tuned to the $D_{1}$ transition of $\mathrm{Rb}^{87} 5 S_{1 / 2}(F=1)-5 P_{1 / 2}\left(F^{\prime}=2\right)$. Radiation from the second ECDL used as a probe is tuned over the transition. The linearly polarized laser beams are combined by a polarizing beam splitter and then sent into the cell with the atomic rubidium vapor. Both beams are parallel and the diameters of the beams are the same, $1 \mathrm{~mm}$. The glass cell $(l=7.5 \mathrm{~cm})$ with atomic rubidium vapor at room temperature $\left(N_{\mathrm{Rb}} \simeq 10^{10} \mathrm{~cm}^{-3}\right)$ is installed in a magnetic shield. A longitudinal magnetic field is created by a solenoid. The polarization of the pump field is strictly right circular. The probe field is a superposition of (uncorrelated) fields with left and right polarizations and equal optical power (the difference is less then $1 \%$ ). The polarization of the probe field is not defined at any moment of time, and the probe field can be considered as an unpolarized light field. We change the time delay $(\simeq 3 \mathrm{~ns})$ between field components of the probe field with opposite polarizations, and we do not observe any influence on the recorded curves.

The typical Doppler-free resonances are shown in Fig. 3. The resonances are recorded on the transitions of $\mathrm{Rb}^{87}$ for different longitudinal magnetic fields. The power of the pump beam is $0.6 \mathrm{~mW}$; the estimated Rabi frequency is several $\gamma^{\prime} \mathrm{s}(\gamma=2 \pi \times 6 \mathrm{MHz})$. This value of the Rabi frequency satisfies conditions for EIMCA in the $V$ scheme. The probe beam power (total) is less than $10 \%$ of the pump beam.

The transition is chosen because the effect is most pronounced in this case. In the linear regime of propagation, the laser light intensity is very small, and the probe transmission on the transition frequency is near 0.9. Nonlinear variation of absorption induced by the pump beam is near $30 \%$ of linear resonance absorption.

Note that it is difficult to define the real amplitude of Doppler-free resonance at zero detuning. Doppler-free

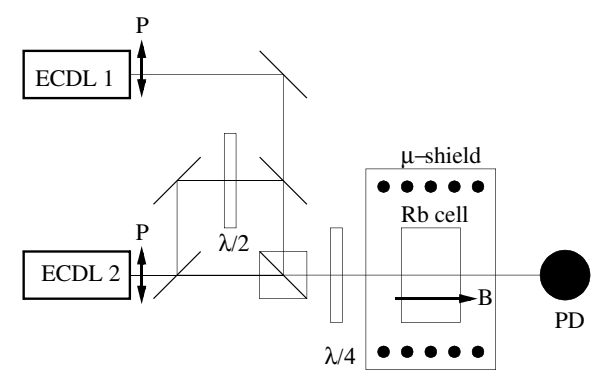

FIG. 2. Experimental setup. 


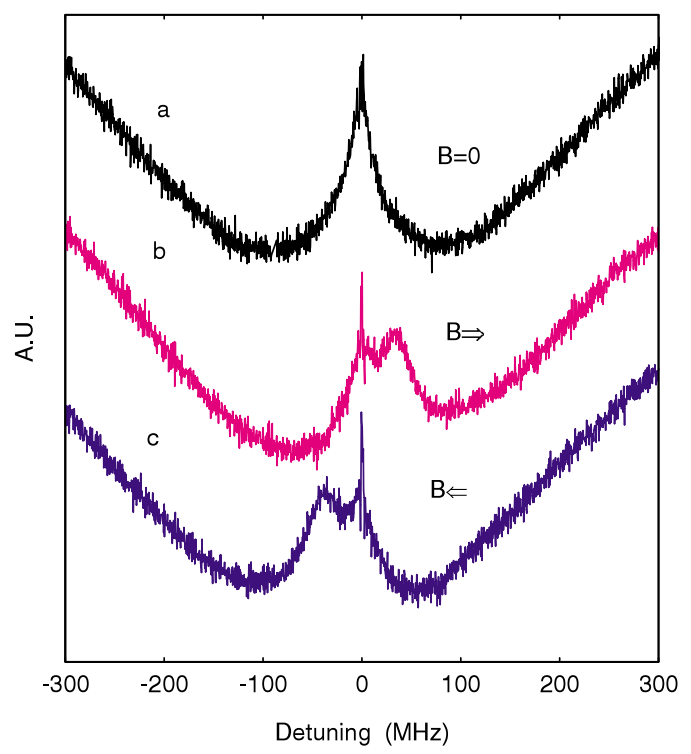

FIG. 3 (color online). Doppler-free resonances on the transition for (a) zero magnetic field $B=0$ and (b),(c) opposite directions of magnetic field $B=77$ Gauss.

resonance on the transition has an additional narrow structure in the center. This narrow structure results from two contributions: one signal is a beat note between the pump and probe fields, and another signal is the electromagnetically induced transparency (EIT) narrow resonance due to ground state coherence. (The beat note at the zero frequency difference and the dark resonance overlap.)

The first curve [Fig. 3(a)] is the resonance on the transition at the zero magnetic field, $B=0$. The other two curves [Figs. 3(b) and 3(c)] are recorded for positive and negative longitudinal magnetic field $B=77$ Gauss (corresponding current is $I=1.4 \mathrm{~A}$, and magnetic field $B$ is related to current $I$ by $B=k I$, where $k=55 \mathrm{G} / \mathrm{A}$ ). Sharp narrow resonances that are combinations of the dark resonance and beat note indicate a zero frequency difference between the pump and probe fields. It is to be noted that the pump laser frequency is tuned to the maximum of linear absorption. One can see that absorption spectra are very different for positive and negative magnetic fields. There is an asymmetry in the absorption coefficient of the resonant atomic medium induced by a circular polarized pump beam.

In Fig. 4, the frequency dependence of absorption difference for the opposite magnetic field is presented [the result of subtraction of curves 3(b) and 3(c) normalized to linear absorption]. To the best of our knowledge, it is the largest EIMCA which has been experimentally observed. Our finding of the giant magnetochiral anisotropy is related to the allowed electric dipole moments of resonance transitions in atoms. For a fixed laser intensity we also studied the dependence of the asymmetry on the magnetic field. The experimentally observed absorption difference as a function of the magnetic field is presented in Fig. 5. One

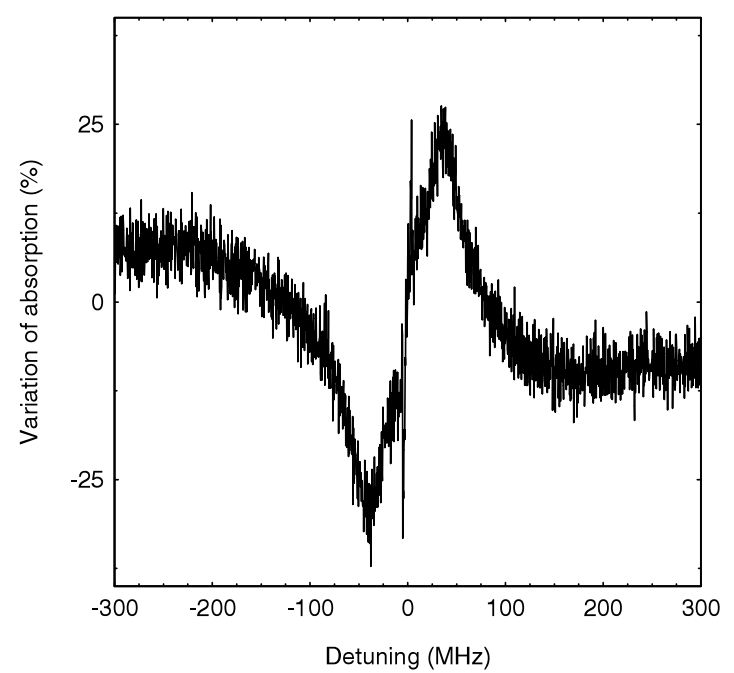

FIG. 4. Relative difference between absorption curves recorded for opposite directions of magnetic field $(B= \pm 77$ Gauss).

can see that for small magnetic fields the asymmetry increases for a range of magnetic fields (where Zeeman splitting is comparable to the resonance width). Once the magnetic field is strong enough to separate resonances, the asymmetry saturates and does not depend on the magnitude of the magnetic field. We observed that above $0.6 \mathrm{~mW}$ of laser pump power the asymmetry changes very little. At $2.4 \mathrm{~mW}$ maximal symmetry is just near $30 \%$.

As one can see, the observed asymmetry is very strong. The effect originates from the different interaction between different polarizations of unpolarized laser light and atomic levels. It can be viewed that one polarization of the probe laser interacts with a different magnetic level of the excited state than the driving laser, forming the so-

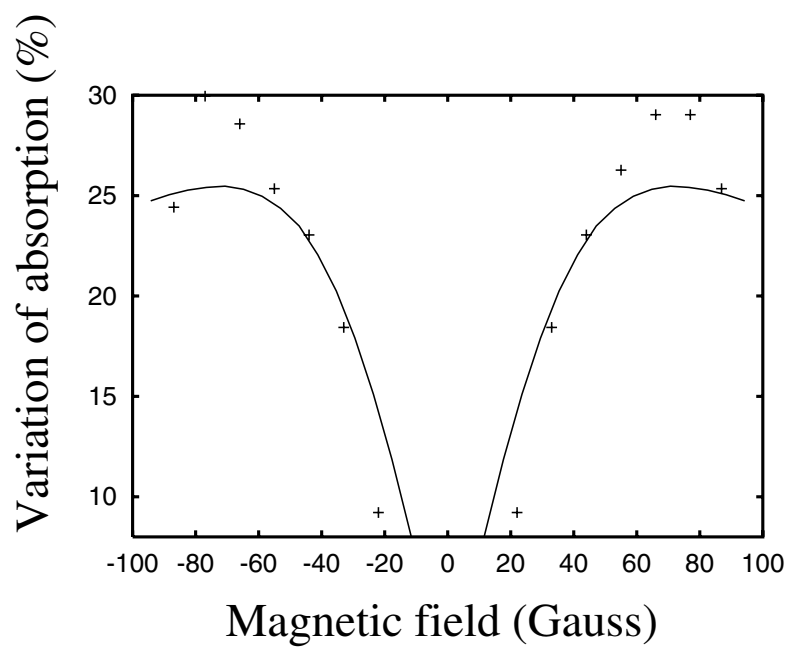

FIG. 5. Crosses: The laser light induced variation of absorption versus magnetic field. Solid line: The calculated laser induced relative variation of absorption versus magnetic field. 
called $V$-type configuration of atomic levels. For this component the EIT conditions can be satisfied, and it travels through the medium without absorption. Meanwhile, the orthogonal polarization of the weak probe field interacts with the same excited level as the driving field does, so the probe field experiences less absorption because of saturation of the transition by the driving field. These two mechanism are different, and they create substantially different responses for different polarizations, leading to chirality.

To answer the question of whether the asymmetry observed in the experiment is close to the maximal theoretical limit, we perform numerical simulations of the selfconsistent set of density matrix and Maxwell equations given by

$$
\frac{\partial \rho}{\partial \tau}=-\frac{i}{\hbar}[H, \rho]-\frac{1}{2}(\Gamma \rho+\rho \Gamma), \quad \frac{\partial \Omega_{i j}}{\partial z}=-i \eta \rho_{i j}(z, \tau),
$$

where $\Gamma$ is the decay operator, and $\rho$ is the atomic density matrix; $\Omega_{i j}$ is the Rabi frequency of the field frequency $\nu$ which is coupled to the transition $i \leftrightarrow j ; \rho_{i j}$ and $\wp_{i j}$ are the coherence and the dipole moment between levels $i$ and $j$ correspondingly; $\eta=\nu N \wp_{i j} /\left(2 \epsilon_{0} c\right) ; N$ is the atomic density; $\epsilon_{0}$ is the permittivity of the vacuum; and $c$ is the speed of light in vacuum. It is instructive to analyze $\chi(\omega)=$ $\eta \gamma(\omega-i \gamma) /\left[(\gamma+i \omega)(\gamma+i k v+i \omega)+|\Omega|^{2}\right]$, where $\omega=\omega_{+f}+g B-\nu ; \gamma$ is the relaxation rate for optical coherences. $\chi^{\prime \prime}=\frac{\eta \gamma^{2}}{|\Omega|^{2}}+\frac{3 \eta \gamma^{2} \omega^{2}}{|\Omega|^{4}}+\frac{2 \eta \gamma^{2} \omega k v}{|\Omega|^{4}}$, where one can see that it is the spatial dispersion term $k v$ (Doppler shift due to atomic motion) that leads to magnetochirality in the $\mathrm{Rb}$ vapor.

We have performed theoretical simulations by using the experimental parameters, and the results of simulations are presented in Fig. 5, where the dependence on magnetic field is shown. One can see good agreement between the simulation and the experiment. The calculated asymmetry is higher for smaller magnetic fields because the $\mathrm{Rb}$ atom has more levels (three sets of $V$ schemes originated from the sublevels of the ground state $F=1, m=0, \pm 1$.

There are various applications of the obtained results ranging from optics to chemistry and biology. For example, the effect of large chirality can be used to create an optically nonisotropic environment, which results in different rates of chemical photoreactions with different enantiomers.

In summary, we have implemented optically induced chirality in the $\mathrm{Rb}$ vapor cell. The observed anisotropy is huge. It is about $30 \%$, and one has the possibility of creating a chiral environment for optical control of chemical reactions involving one type of enantiomer. Another interesting possibility is to observe an inverse effect to the one demonstrated here.

We thank D. Budker, J. Dowling, P. Hemmer, N. Kalugin, and A. Muthukrishnan for useful and fruitful discussions and gratefully acknowledge the support from the Office of Naval Research, the Air Force Research Laboratory (Rome, NY), Defense Advanced Research Projects Agency-QuIST, Texas A\&M University Telecommunication and Information Task Force (TITF) Initiative, the NSF, and the Welch Foundation (Grant No. A1261).

*On leave from Physical Research Laboratory, Navrangpura, Ahmedabad-380 009, India.

[1] S.E. Harris, G. Y. Yin, M. Jain, and A. J. Meriam, Phil. Trans. R. Soc. A 355, 2291 (1997).

[2] S. P. Tewari and G. S. Agarwal, Phys. Rev. Lett. 56, 1811 (1986).

[3] A. B. Matsko, O. Kocharovskaya, Y. Rostovtsev, G. R. Welch, A.S. Zibrov, and M. O. Scully, in Advances in Atomic, Molecular, and Optical Physics, edited by B. Bederson and H. Walther (Academic Press, San Diego, CA, 2001), Vol. 46, p. 191.

[4] K. Hakuta, L. Marmet, and B. P. Stoicheff, Phys. Rev. A 45, 5152 (1992).

[5] S. E. Harris and Y. Yamamoto, Phys. Rev. Lett. 81, 3611 (1998).

[6] L. V. Hau, S.E. Harris, Z. Dutton, and C. H. Behroozi, Nature (London) 397, 594 (1999).

[7] M. M. Kash, V. A. Sautenkov, A. S. Zibrov, L. Hollberg, G. R. Welch, M. D. Lukin, Y. Rostovtsev, E. S. Fry, and M. O. Scully, Phys. Rev. Lett. 82, 5229 (1999).

[8] D. Budker, D.F. Kimball, S. M. Rochester, and V. V. Yaschuk, Phys. Rev. Lett. 83, 1767 (1999).

[9] D. F. Phillips, A. Fleschhauer, A. Mair, R. L. Walsworth, and M. D. Lukin, Phys. Rev. Lett. 86, 783 (2001); M. Bajcsy, A.S. Zibrov, and M.D. Lukin, Nature (London) 426, 638 (2003).

[10] A.S. Zibrov, A. V. Matsko, O. Kocharovskaya, Y. Rostovtsev, G. R. Welch, and M. O. Scully, Phys. Rev. Lett. 88, 103601 (2002).

[11] L. D. Landau, E. M. Lifshitz, and L.P. Pitaevski, Electrodynamics of Continuous Media (Pergamon, Oxford, 1984).

[12] V. A. Markelov, M. A. Novikov, and A. A. Turkin, JETP Lett. 25, 378 (1977).

[13] N. B. Baranova and B. Ya. Zel'dovich, Mol. Phys. 38, 1085 (1979).

[14] L. D. Barron, Nature (London) 405, 895 (2000); L. D. Barron and J. Vrbancich, Mol. Phys. 51, 715 (1984).

[15] G. L. J. A. Rikken and E. Raupach, Nature (London) 390, 493 (1997).

[16] N. G. Kalugin, P. Kleindienst, and G. H. Wagniere, Chem. Phys. 248, 105 (1999).

[17] M. Vallet, R. Ghosh, A. Le Floch, T. Ruchon, F. Bretenaker, and J.-Y. Thepot, Phys. Rev. Lett. 87, 183003 (2001).

[18] G. S. Agarwal and S. Dasgupta, Phys. Rev. A 67, 023814 (2003).

[19] S. Wielandy and A. L. Gaeta, Phys. Rev. Lett. 81, 3359 (1998). 